\title{
Existence Theory of Differential Equations of Arbitrary
}

\author{
Kamal Shah and Yongjin Li \\ Additional information is available at the end of the chapter \\ http://dx.doi.org/10.5772/intechopen.75523
}

\begin{abstract}
The aims of this chapter are devoted to investigate a system of fractional-order differential equations (FDEs) with multipoint boundary conditions. Necessary and sufficient conditions are investigated for at most one solution to the proposed problem. Also, results for the existence of at least one or two positive solutions are developed by using a fixed-point theorem of concave-type operator for the considered problem. Further, we extend the conditions for more than two solutions and established some adequate conditions for multiplicity results to the proposed problem. Also, a result devoted to Hyers-Ulam stability is discussed. Suitable examples are provided to verify the established results.
\end{abstract}

Keywords: fractional differential equations, coupled system, boundary condition, concave operator

Mathematics subject classification: 26A33, 34A08, 35B40

\section{Introduction}

Arbitrary-order differential equations are the excellent tools in the description of many phenomena and process in different fields of science, technology, and engineering (see [1, 2]). Therefore, considerable attention has been paid to the subject of differential equations of arbitrary order (see [3-5] and the references therein). The area devoted to the existence of positive solutions to fractional differential equations and their system especially coupled systems was greatly studied by many authors (for details see [6-9]). In all these articles, the concerned results were obtained by using classical fixed point theorems like Banach contraction principle, Leray-Schauder fixed point theorem, and fixed point theorems of cone type. The aforesaid area has been very well explored for both ordinary- and arbitrary-order differential equations. Existence and uniqueness results for nonlinear and linear, classical, 
as well as arbitrary-order differential equations have been investigated in many papers (see few of them as [10-13]).

Another warm area of research in the theory of fractional-order differential equations (FDEs) is devoted to the multiplicity of solutions. Plenty of research articles are available on this topic in literature. In [14], the author studied the given boundary value problem (BVP) for existence of multiple solutions:

$$
\left\{\begin{array}{l}
\mathscr{D}^{\theta_{1}} p(t)+\mathcal{H}(t, p(t))=0, \quad t \in \mathbf{I}, \quad \theta_{1} \in(1,2], \\
\left.p(t)\right|_{t=0}=\left.p(t)\right|_{t=1}=0 .
\end{array}\right.
$$

where $\mathscr{D}$ is the Riemann-Liouville derivative of non-integer order and $\mathbf{I}=[0,1]$. In same line, Kaufmann and Mboumi [15] studied the given boundary value problem of fractional differential equations for multiplicity of positive solutions:

$$
\left\{\begin{array}{l}
\mathscr{D}^{\theta_{1}} p(t)+\phi(t) \mathcal{H}(t, p(t))=0, \quad t \in \mathbf{I}, \quad \theta_{1} \in(1,2], \\
\left.p(t)\right|_{t=0}=\left.p^{\prime}(t)\right|_{t=1}=0
\end{array}\right.
$$

where $\mathscr{D}$ is the Riemann-Liouville derivative and $\phi \in C(\mathbf{I}, \mathbf{R}), \quad \mathcal{H} \in C(\mathbf{I} \times \mathbf{R}, \mathbf{R})$.

In the last few decades, the theory devoted to the multiplicity of solutions is very well extended to coupled systems of nonlinear FDEs, and we refer to few papers in [16-18]. Wang et al. [19] established some conditions under which the given system of three point BVP

$$
\left\{\begin{array}{l}
\mathscr{D}^{\theta_{1}} p(t)=\mathcal{H}_{1}(t, q(t)) ; \quad t \in \mathbf{I}, \\
\mathscr{D}^{\theta_{2}} q(t)=\mathcal{H}_{2}(t, p(t)) ; \quad t \in \mathbf{I}, \\
p(t)_{t=0}=0, \quad p(t)_{t=1}=\left.\mu p(t)\right|_{t=\xi},\left.q(t)\right|_{t=0}=0, \quad q(t)_{t=1}=\left.v q(t)\right|_{t=\xi^{\prime}}
\end{array}\right.
$$

has a solution, where $\theta_{1}, \theta_{2} \in(1,2]$ and $\mu, v \in \mathbf{I}, \xi \in(0,1), \mathcal{H}_{i}:[0,1] \times \mathbf{R} \rightarrow \mathbf{R}$ for $i=1,2$ are nonlinear functions.

In the last few decades, another important aspect devoted to stability analysis of FDEs with initial/boundary conditions has been given much attention. This is because stability is very important from the numerical and optimization point of view. Various forms of stabilities were studied for the aforesaid FDEs including exponential, Mittag-Leffler, and Lyapunov stability. Recently, Hyers-Ulam stability has given more attention. This concept was initially introduced by Ulam and then by Hyers (for details see [20-22]). Now, many articles have been written on this concept (see [23-27]). So far, the aforementioned stability has not yet well studied for multipoint BVPs of FDEs. Motivated by the aforesaid discussion, we propose the following coupled system of four-point BVP provided as

$$
\left\{\begin{array}{l}
\mathscr{D}^{\theta_{1}} p(t)=\mathcal{H}_{1}(t, p(t), q(t)) ; \quad t \in \mathbf{I} ; \quad \theta_{1} \in(m-1, m], \\
\mathscr{D}^{\theta_{2}} q(t)=\mathcal{H}_{2}(t, p(t), q(t)) ; \quad t \in \mathbf{I} ; \quad \theta_{2} \in(m-1, m], \\
p^{(j)}(t)_{t=0}=\left.q^{(j)}(t)\right|_{t=0}=0,\left.\quad p(t)\right|_{t=1}=\left.\left.p(t)\right|_{t=\eta^{\prime}} \quad q(t)\right|_{t=1}=\left.q(t)\right|_{t=\xi} .
\end{array}\right.
$$


where $j=0,1,2, \cdots m-2, \quad m \geq 3, \quad \mathbf{I}=[0,1], \quad \eta, \xi \in(0,1), \mathcal{H}_{1}, \mathcal{H}_{2}:[0,1] \times\{0\} \cup \mathbf{R}^{+} \times\{0\} \cup \mathbf{R}^{+} \rightarrow$ $\{0\} \cup \mathbf{R}^{+}$are continuous functions, and $\mathscr{D}^{\theta_{1}}, \mathscr{D}^{\theta_{2}}$ stand for Riemann-Liouville fractional derivative of order $\theta_{1}, \theta_{2}$ in sequel. We obtain necessary and sufficient conditions for the existence of solution to system (1) by using another type of fixed point result based on a concave-type operator with increasing or decreasing property. The idea then extends to form some conditions which ensure multiplicity of solutions to the considered problem. Also, we discuss some results about the HyersUlam stability for the considered problem. Further by providing examples, we illustrate the established results.

\section{Preliminaries}

In the current section, we review few fundamental lemmas and results found in $[2,4,6,28,29]$.

Definition 2.1. Arbitrary-order integral of function $\psi:(0, \infty) \rightarrow \mathbf{R}$ is recalled as

$$
\mathcal{I}^{\theta_{1}} \psi(t)=\frac{1}{\Gamma\left(\theta_{1}\right)} \int_{0}^{t}(t-s)^{\theta_{1}-1} \psi(s) d s
$$

where $\theta_{1}>0$ is a real number and also the integral is pointwise defined on $\mathbf{R}^{+}$

Definition 2.2. Arbitrary-order derivative in Riemann-Liouville sense for a function $\psi \in((0, \infty), \mathbf{R})$ is given by

$$
\mathscr{D}^{\theta_{1}} \psi(t)=\left(\frac{d}{d t}\right)^{m} \int_{0}^{t} \frac{(t-s)^{m-\theta_{1}-1}}{\Gamma\left(m-\theta_{1}\right)} \psi(s) d s, \theta_{1}>0, \text { where } m=\left[\theta_{1}\right]+1
$$

Lemma 2.3. [16] Let $\theta_{1}>0$, then for arbitrary $C_{j} \in \mathbf{R}, j=1,2, \ldots, m, m=\left[\theta_{1}\right]+1$, and the solution of

$$
\mathscr{D}^{\theta_{1}} \psi(t)=f(t)
$$

is provided by

$$
\psi(t)=\mathcal{I}^{\theta_{1}} f(t)+C_{1} t^{\theta_{1}-1}+C_{2} t^{\theta_{1}-2}+\ldots+C_{m} t^{\theta_{1}-m}
$$

Definition 2.4. $[17,28]$ Consider a Banach space $\mathbf{E}$ with a closed set $\mathbf{C} \subset \mathbf{E}$. Then, $\mathbf{C}$ is said to be partially ordered if $p \preceq q$ such that $q-p \in \mathbf{C}$. Further, $\mathbf{C}$ is said to be a cone if it holds the given conditions:

1. $p \in \mathbf{C}$ and for a real constant $\kappa \geq 0$ the relation $\kappa p \in \mathbf{C}$ holds.

2. $p$ and $-p \in \mathbf{C}$ yield that $0 \in \mathbf{C}$, where 0 is zero element of Banach space $\mathbf{E}$ 
Definition 2.5. $[17,28]$ A closed and convex set $\mathbf{C}$ of $\mathbf{E}$ is said to be a normal cone if it obeys the given properties:

1. For $0 \preceq p \preceq q \in \mathbf{E}$, there exists $\beta>0$, such that $\|p\|_{\mathrm{E}} \leq \beta\|q\|_{\mathrm{E}}$;

2. $p \sim q$, for all $p, q \in \mathrm{E}$ yields that there exist constants $a, b>0$ such that $a p \preceq q \leq b q$.

Remark 2.6. As $\sim$ is an equivalence relation, therefore defines a set $\mathbf{C}_{f}=\{p \in \mathbf{E}: p \sim f\}$ for $f \in \mathbf{C}$. Obviously, one can derive that $\mathbf{C}_{f} \subset \mathbf{C}$ for $f>0$.

Definition 2.7. The operator $\mathcal{S}: \mathbf{C} \rightarrow \mathbf{C}$ is said to be $\lambda$ concave for every $\theta, \lambda \in(0,1), p \in \mathbf{C}$, if and only if $\mathcal{S}(\lambda p) \succeq \theta^{\lambda} \mathcal{S} p$.

Definition 2.8. The operator $\mathcal{S}: \mathbf{C} \rightarrow \mathbf{C}$ is said to be to be increasing if $p, q \in \mathbf{C}, p \preceq q$ gives that $\mathcal{S} p \preceq \mathcal{S} q$.

Lemma 2.9. $[17,28]$ Assume that $\mathcal{S}: \mathbf{C} \rightarrow \mathbf{C}$ is increasing $\lambda$-concave operator for a normal cone $\mathbf{C}$ produced by Banach space $\mathbf{E}$, such that there exists $p>0$ with $\mathcal{S} f \in \mathbf{C}_{f}$. Then, $\mathcal{S}$ has a unique fixed point $p \in \mathbf{C}_{f}$

Theorem 2.10. [30] Let $\mathrm{E}$ be a Banach space with $\mathrm{C} \subseteq \mathcal{B}$, which is closed and convex. Let $\mathcal{E}$ be a relatively open subset of $\mathbf{C}$ with $0 \in \mathcal{E}$ and $\mathcal{S}: \overline{\mathcal{E}} \rightarrow \mathbf{C}$ be a continuous and compact operator. Then.

1. The operator $\mathcal{S}$ has a fixed point in $\overline{\mathcal{E}}$,

2. There exist $w \in \partial \mathscr{E}$ and $\lambda \in(0,1)$ with $w=\lambda \mathcal{S} w$.

Lemma 2.11. [30] For a Banach space $\mathbf{E}$ together with a cone $\mathbf{C}$, there exist two relatively open subsets $\mathbf{A}_{1}$ and $\mathbf{A}_{2}$ of $\mathbf{E}$ such that $0 \in \mathbf{A}_{1} \subset \overline{\mathbf{A}}_{1} \subset \mathbf{A}_{2}$. Moreover, for a completely continuous operator $\mathcal{S}: \mathbf{C} \cap\left(\overline{\mathbf{A}}_{2} \backslash \mathbf{A}_{1}\right) \rightarrow \mathbf{C}$, one of the given conditions holds:

1. $\|\mathcal{S} p\| \leq\|p\|$ for all $p \in \mathbf{C} \cap \partial \mathbf{A}_{1} ; \quad\|\mathcal{S} p\| \geq\|p\|$, for all $p \in \mathbf{C} \cap \partial \mathbf{A}_{2}$;

2. $\|\mathcal{S} p\| \geq\|p\|$ for all $p \in \mathbf{C} \cap \partial \mathbf{A}_{1} ; \quad\|\mathcal{S} p\| \leq\|p\|$, for all $p \in \mathbf{C} \cap \partial \mathbf{A}_{2}$

Then, $\mathcal{S}$ has at least one fixed point in $\mathbf{C} \cap\left(\overline{\mathbf{A}}_{2} \backslash \mathbf{A}_{1}\right)$.

\section{Main results}

Theorem 3.1. Let $\varphi \in C([0,1], \mathbf{R}), \eta \in(0,1)$ and $\lambda_{1}=1-\eta^{\theta_{1}-1}<1$, and then the unique solution to $B V P$ of linear FDE

$$
\left\{\begin{array}{l}
\mathscr{D}^{\theta_{1}} p(t)=\varphi(t), t \in \mathbf{I}, \quad \theta_{1} \in(m-1, m] \\
p^{(j)}(t)_{t=0}=0,\left.p(t)\right|_{t=1}=\left.p(t)\right|_{t=\eta^{\prime}} j=0,1,2, \cdots m-2, \quad m \geq 3
\end{array}\right.
$$

is given by

$$
p(t)=\int_{0}^{1} \mathbf{G}(t, s) \varphi(s) d s,
$$


where $\mathbf{G}(t, s)$ is the Green's function defined by

$$
\mathbf{G}(t, s)=\frac{1}{\Gamma\left(\theta_{1}\right)}\left\{\begin{array}{l}
\frac{1}{\lambda_{1}}\left[-[t(1-s)]^{\theta_{1}-1}+[t(\eta-s)]^{\theta_{1}-1}\right]+(t-s)^{\theta_{1}-1}, 0 \leq s \leq t \leq \eta \leq 1, \\
\lambda_{1}\left[-[t(1-s)]^{\theta_{1}-1}+[t(\eta-s)]^{\theta_{1}-1}\right], 0 \leq t \leq s \leq \eta \leq 1, \\
-\frac{1}{\lambda_{1}}[t(1-s)]^{\theta_{1}-1}+(t-s)^{\theta_{1}-1}, 0 \leq \eta \leq s \leq t \leq 1, \\
-\frac{1}{\lambda_{1}}[t(1-s)]^{\theta_{1}-1}, 0 \leq \eta \leq t \leq s \leq 1 .
\end{array}\right.
$$

Proof. In view of Lemma 2.3, we may write Eq. (2) as

$$
p(t)=\mathcal{I}^{\theta_{1}} \varphi(t)+C_{1} t^{\theta_{1}-1}+C_{2} t^{\theta_{1}-2}+\ldots+C_{m} t^{\theta_{1}-m} .
$$

In view of conditions $p^{(j)}(t)_{t=0}=0, j=0,1, \ldots m-2, m \geq 3$, Eq. (5) suffers from singularity; therefore, we have $C_{2}=C_{3}=\ldots=C_{n}=0$. Hence, Eq. (5) becomes

$$
p(t)=\mathcal{I}^{\theta_{1}} \varphi(t)+C_{1} t^{\theta_{1}-1} .
$$

Applying boundary condition $\left.p(t)\right|_{t=1}=\left.p(t)\right|_{t=\eta}$ and $d=1-\eta_{1}^{\theta}$ in Eq. (6), one has

$$
\begin{aligned}
& p(t)=\mathcal{I}^{\theta_{1}} \varphi(t)+\frac{t^{\theta_{1}-1}}{\lambda_{1}}\left[\mathcal{I}^{\theta_{1}} \varphi(\eta)-\mathcal{I}^{\theta_{1}} \varphi(1)\right] \\
& p(t)=\int_{0}^{1} \mathbf{G}(t, s) \varphi(s) d s .
\end{aligned}
$$

where $\mathbf{G}(t, s)$ is Green's function given in Eq. (4).

In view of Theorem 3.1 and using $\lambda_{1}=1-\eta^{\theta_{1}-1}, \lambda_{2}=1-\xi^{\theta_{2}-1}$, the corresponding coupled system of integral equations to the proposed system (1) is given as

$$
\left\{\begin{array}{l}
p(t)=\int_{0}^{1} \mathbf{G}_{1}(t, s) \mathcal{H}_{1}(s, p(s), q(s)) d s, \\
q(t)=\int_{0}^{1} \mathbf{G}_{2}(t, s) \mathcal{H}_{2}(s, p(s), q(s)) d s,
\end{array}\right.
$$

where $\mathbf{G}_{1}(t, s), \mathbf{G}_{2}(t, s)$ are Green's functions, which can be similarly computed like in Theorem 3.1. Further, they are continuous on $\mathbf{I} \times \mathbf{I}$ and satisfy the following properties:

i. $\max _{t \in \mathbf{I}}\left|\mathbf{G}_{1}(t, s)\right| \leq \frac{\left(\lambda_{1}+1\right)(1-s)^{\theta_{1}-1}}{\lambda_{1}}=\mathbf{G}_{1}(1, s)$, for all $s \in \mathbf{I}$, $\max _{t \in \mathbf{I}}\left|\mathbf{G}_{1}(t, s)\right| \leq \frac{\left(\lambda_{2}+1\right)(1-s)^{\theta_{2}-1}}{\lambda_{2}}=\mathbf{G}_{2}(1, s)$, for all $s \in \mathbf{I}$;

ii. $\min _{t \in[\theta, 1-\theta]} \mathbf{G}_{1}(t, s) \geq \frac{\gamma_{1}(s)}{2} \mathbf{G}(1, s)$ for every $\theta s \in(0,1)$; $\min _{t \in[\theta, 1-\theta]} \mathbf{G}_{2}(t, s) \geq \frac{\gamma_{2}(s)}{2} \mathbf{G}(1, s)$ for every $\theta s \in(0,1)$; Further, taking that $\gamma=\inf \left\{\gamma_{1}=\theta^{\theta_{1}-1}, \gamma_{2}=\theta^{\theta_{2}-1}\right\}$. 
Let us define a Banach space by $\mathbf{E}=\{p(t) \mid p \in C(\mathbf{I})\}$ endowed with a norm $\|p\|_{\mathbf{E}}=\max _{t \in \mathbf{I}}|p(t)|$. Further, in the norm for the product space, we define it as $\|(p, q)\|_{\mathbf{E} \times \mathbf{E}}=\|p\|_{\mathbf{E}}+\|q\|_{\mathbf{E}}$. Clearly, $\left(\mathbf{E} \times \mathbf{E},\|\cdot\|_{\mathbf{E} \times \mathbf{E}}\right)$ is a Banach space. Onward, we define the cone $\mathbf{C} \subset \mathbf{E} \times \mathbf{E}$ by

$$
\mathbf{C}=\left\{(p, q) \in \mathbf{E} \times \mathbf{E}: \min _{t \in \mathbf{I}}[p(t)+q(t)] \geq \gamma\|(p, q)\|_{\mathbf{E} \times \mathbf{E}}\right\} .
$$

Consider an operator $\mathcal{S}: \mathbf{E} \times \mathbf{E} \rightarrow \mathbf{E} \times \mathbf{E}$ defined by

$$
\begin{aligned}
\mathcal{S}(p, q)(t) & =\left(\int_{0}^{1} \mathbf{G}_{1}(t, s) \mathcal{H}_{1}(s, p(s), q(s)) d s, \int_{0}^{1} \mathbf{G}_{2}(t, s) \mathcal{H}_{2}(s, p(s), q(s)) d s\right) . \\
& =\left(\mathcal{S}_{1} p(t), \mathcal{S}_{2} q(t)\right) .
\end{aligned}
$$

It is to be noted that the fixed points of the operator $\mathcal{S}$ correspond with the solution of the system (1) under consideration.

Theorem 3.2. Under the continuity of $\mathcal{H}_{1}, \mathcal{H}_{2}: \mathbf{I} \times \mathbf{R}^{+} \cup\{0\} \times \mathbf{R}^{+} \cup\{0\} \rightarrow \mathbf{R}^{+}\{0\}$, the operator $\mathcal{S}$ satisfies that $\mathcal{S}(\mathbf{C}) \subset \mathbf{C}$ and $\mathcal{S}: \mathbf{C} \rightarrow \mathbf{C}$ is completely continuous.

Proof. To derive $\mathcal{S}(\mathbf{C}) \subset \mathbf{C}$, let $(p, q) \in \mathbf{C}$, and then we have

$$
\mathcal{S}_{1}(p(t), q(t))=\int_{0}^{1} \mathbf{G}_{1}(t, s) \mathcal{H}_{1}(s, p(s), q(s)) d s \geq \gamma_{1} \int_{0}^{1} \mathbf{G}_{1}(1, s) \mathcal{H}_{1}(s, p(s)), q(s) d s .
$$

Also, we get

$$
\mathcal{S}_{1}(p(t), q(t))=\int_{0}^{1} \mathbf{G}_{1}(t, s) \mathcal{H}_{1}(s, p(s), q(s)) d s \leq \int_{0}^{1} \mathbf{G}_{1}(1, s) \mathcal{H}_{1}(s, p(s)), q(s) d s .
$$

Thus, from Eqs. (10) and (11), we have

$$
\mathcal{S}_{1}(p(t), q(t)) \geq \gamma\left\|\mathcal{S}_{1}(p, q)\right\|_{\mathbf{E}^{\prime}} \text { for every } t \in \mathbf{I} .
$$

Similarly, we can obtain

$$
\mathcal{S}_{2}(p(t), q(t)) \geq \gamma\left\|\mathcal{S}_{2}(p, q)\right\|_{\mathrm{E}} \text {, for every } t \in \mathbf{I} .
$$

Thus $\mathcal{S}_{1}(p(t), q(t))+\mathcal{S}_{2}(p(t), q(t)) \geq \gamma\|(p, q)\|_{\mathbf{E} \times \mathbf{E}}$, for all $t \in \mathbf{I}$,

$$
\min _{t \in \mathbf{I}}\left[\mathcal{S}_{1}(p(t), q(t))+\mathcal{S}_{2}(p(t), q(t))\right] \geq \gamma\|(p, q)\|_{\mathbf{E} \times \mathbf{E}} .
$$

Hence, we have $\mathcal{S}(p, q) \in \mathbf{C} \Rightarrow \mathcal{S}(\mathbf{C}) \subset \mathbf{C}$. 
Let us consider

$$
\max _{t \in \mathbf{I}}\left|\mathcal{H}_{1}(t, p(t), q(t))\right| \leq \mathcal{M}_{1}, \quad \max _{t \in \mathbf{I}}\left|\mathcal{H}_{2}(t, p(t), q(t))\right| \leq \mathcal{M}_{2}
$$

Then, we consider $t_{1}<t_{2} \in \mathbf{I}$, such that

$$
\begin{aligned}
\left|\mathcal{S}_{1}(p, q)\left(t_{2}\right)-\mathcal{S}_{1}(p, q)\left(t_{1}\right)\right|= & \left|\int_{0}^{1}\left(\mathbf{G}\left(t_{2}, s\right)-\mathbf{G}_{1}\left(t_{1}, s\right)\right) \mathcal{H}_{1}(s, p(s), q(s)) d s\right| \\
& \leq \frac{\mathcal{M}_{1}}{\Gamma\left(\theta_{1}\right)}\left[\frac{\left(t_{2}^{\theta_{1}-1}-t_{1}^{\theta_{1}-1}\right)}{\lambda_{1}}\left(\int_{0}^{\eta}(\eta-s)^{\theta_{1}-1} d s-\int_{0}^{1}(1-s)^{\theta_{1}-1} d s\right)\right] \\
& +\frac{\mathcal{M}_{1}}{\Gamma\left(\theta_{1}\right)}\left[\int_{0}^{t_{2}}\left(t_{2}-s\right)^{\theta_{1}-1} d s-\int_{0}^{t_{1}}\left(t_{1}-s\right)^{\theta_{1}-1} d s\right] \\
& \leq \frac{\mathcal{M}_{1}}{\lambda_{1} \Gamma\left(\theta_{1}+1\right)}\left[\left(t_{2}^{\theta_{1}-1}-t_{1}^{\theta_{1}-1}\right)\left(\eta^{\theta_{1}}-\lambda_{1}\right)+\lambda_{1}\left(t_{2}^{\theta_{1}}-t_{1}^{\theta_{1}}\right)\right] .
\end{aligned}
$$

By the same fashion, we obtain for $\mathcal{S}_{2}$ as

$$
\left|\mathcal{S}_{2}(p, q)\left(t_{2}\right)-\mathcal{S}_{2}(p, q)\left(t_{1}\right)\right| \leq \frac{\mathcal{M}_{2}}{\lambda_{2} \Gamma\left(\theta_{2}+1\right)}\left[\left(t_{2}^{\theta_{2}-1}-t_{1}^{\theta_{2}-1}\right)\left(\xi^{\theta_{2}}-\lambda_{2}\right)+\lambda_{2}\left(t_{2}^{\theta_{2}}-t_{1}^{\theta_{2}}\right)\right] .
$$

The right hand sides of Eqs. (12) and (13) are approaching to zero at $t_{1} \rightarrow t_{2}$. Thus, the operator $\mathcal{S}$ is equi-continuous. Therefore, thanks to the Arzelá-Ascoli theorem, we receive that $\mathcal{S}=\left(\mathcal{S}_{1}, \mathcal{S}_{2}\right): \mathbf{C} \rightarrow \mathrm{C}$ is completely continuous.

Theorem 3.3. Due to continuity of $\mathcal{H}_{1}$ and $\mathcal{H}_{2}$ on $\mathbf{I} \times \mathbf{R}^{+} \cup\{0\} \times \mathbf{R}^{+} \cup\{0\} \rightarrow \mathbf{R}^{+}$, there exist $\varphi_{j}, \psi_{j}, \sigma_{j}(j=1,2):(0,1) \rightarrow \mathbf{R}^{+} \cup\{0\}$ for $t \in(0,1), \quad p, \quad q \geq 0$ such that

$$
\begin{aligned}
\left|\mathcal{H}_{1}(t, p(t), q(t))\right| & \leq \varphi_{1}(t)+\psi_{1}(t)|p(t)|+\sigma_{1}(t)|q(t)| \\
\left|\mathcal{H}_{2}(t, p(t), q(t))\right| & \leq \varphi_{2}(t)+\psi_{2}(t)|p(t)|+\sigma_{2}(t)|q(t)|
\end{aligned}
$$

along with the following conditions:

$$
\begin{array}{ll}
\text { i. } & \Delta_{1}=\int_{0}^{1} \mathbf{G}_{1}(1, s) \varphi_{1}(s) d s<\infty, \quad \Lambda_{1}=\int_{0}^{1} \mathbf{G}_{1}(1, s)\left[\psi_{1}(s)+\sigma_{1}(s)\right] d s<1 ; \\
\text { ii. } & \Delta_{2}=\int_{0}^{1} \mathbf{G}_{2}(1, s) \varphi_{2}(s) d s<\infty, \Lambda_{2}=\int_{0}^{1} \mathbf{G}_{2}(1, s)\left[\psi_{2}(s)+\sigma_{2}(s)\right] d s<1
\end{array}
$$

are satisfied. Then, the system (1) has at least one solution $(p, q)$ which lies in

$$
\mathcal{E}=\left\{(p, q) \in \mathbf{C}:\|(p, q)\|_{\mathbf{E} \times \mathbf{E}}<\min \left(\frac{2 \Delta_{1}}{1-2 \Lambda_{1}}, \frac{2 \Delta_{2}}{1-2 \Lambda_{2}}\right)\right\} .
$$


Proof. Let $\mathcal{E}=\left\{(p, q) \in \mathbf{C}:\|(p, q)\|_{\mathrm{E} \times \mathbf{E}}<r\right\}$ with $\min \left(\frac{2 \Delta_{1}}{1-2 \Lambda_{1}}, \frac{2 \Delta_{2}}{1-2 \Lambda_{2}}\right)<r$.

Define the operator $\mathcal{S}: \overline{\mathcal{E}} \rightarrow \mathbf{C}$ as in Eq. (9).

Let $(p, q) \in \mathcal{E}$ that is $\|(p, q)\|_{\mathbf{E} \times \mathbf{E}}<r$. Then, we have

$$
\begin{aligned}
\left|\mathcal{S}_{1}(p, q)(t)\right| & =\max _{t \in \mathbf{I}}\left|\int_{0}^{1} \mathbf{G}_{1}(t, s) \mathcal{H}_{1}(s, p(s), q(s)) d s\right| \\
& \leq\left(\int_{0}^{1} \mathbf{G}_{1}(1, s) \varphi_{1}(s) d s+\int_{0}^{1} \mathbf{G}_{1}(1, s) \psi_{1}(s)|p(s)| d s+\int_{0}^{1} \mathbf{G}_{1}(1, s) \sigma_{1}(s)|q(s)| d s\right) \\
& \leq \int_{0}^{1} \mathbf{G}_{1}(1, s) \varphi_{1}(s) d s+r\left[\int_{0}^{1} \mathbf{G}_{1}(1, s)\left[\psi_{1}(s)+\sigma_{1}(s)\right] d s\right]=\Delta_{1}+r \Lambda_{1} \leq \frac{r}{2} .
\end{aligned}
$$

Thus, from Eq. (14), we have

$$
\left\|\mathcal{S}_{1}(p, q)\right\|_{\mathrm{E}} \leq \frac{r}{2}
$$

Similarly, one can derive that

$$
\left\|\mathcal{S}_{2}(p, q)\right\|_{\mathrm{E}} \leq \frac{r}{2}
$$

Thus, from Eqs. (15) and (16), we get

$$
\|\mathcal{S}(p, q)\|_{\mathrm{E} \times \mathbf{E}} \leq r
$$

Therefore, $\mathcal{S}(p, q) \subseteq \overline{\mathcal{E}}$. Hence, by Theorem 3.2 the operator $\mathcal{S}: \overline{\mathcal{E}} \rightarrow \mathcal{E}$ is completely continuous. Consider the eigenvalue problem:

$$
(p, q)=\rho \mathcal{S}(p, q), \text { with } \rho \in(0,1)
$$

Under the assumption that $(p, q)$ is a solution of Eq. (18) for $\rho \in(0,1)$, we have

$$
\begin{aligned}
& \begin{array}{l}
|p(t)| \leq \rho \max _{t \in \mathbf{I}} \int_{0}^{1} \mathbf{G}_{1}(t, s)\left|\mathcal{H}_{1}(s, p(s), q(s)) d s\right| \\
\quad \leq \rho\left[\int_{0}^{1} \mathrm{G}_{1}(1, s) \varphi_{1}(s) d s+\int_{0}^{1} \mathrm{G}_{1}(1, s)\left(\psi_{1}(s)|p(s)|+\sigma_{1}(s)|q(s)|\right) d s\right] \\
\quad \leq \rho\left(\Delta_{1}+r \Lambda_{1}\right)
\end{array} \\
& \text { which implies that }\|p\|_{\mathrm{E}}<\frac{r}{2} .
\end{aligned}
$$

Similarly, we can obtain that $\|q\|_{\mathrm{E}}<\frac{r}{2}$, so $\|(p, q)\|_{\mathrm{E} \times \mathbf{E}}<r$, which implies that $(p, q)$ does not belong to $\partial \mathcal{E}$ for all $\rho \in(0,1)$. Therefore, due to Theorem $2.10, \mathcal{S}$ has a fixed point in $\overline{\mathcal{E}}$ 
Assume that the given hypothesis holds:

$\left(H_{1}\right)$ The nonlinear functions $\mathcal{H}_{1}$ and $\mathcal{H}_{2}$ are continuous on $\mathbf{I} \times \mathbf{R}^{+} \cup\{0\} \times \mathbf{R}^{+} \cup\{0\} \rightarrow \mathbf{R}^{+} \cup\{0\}$

$\left(H_{2}\right)$ For all $t \in \mathbf{I}$, we have

$$
\mathcal{H}_{1}(t, p, q)=\neq 0, \quad \mathcal{H}_{2}(t, p, q) \neq 0, \text { at }(p, q)=(0,0)
$$

and

$$
\mathcal{H}_{1}(t, p, q) \neq 1, \quad \mathcal{H}_{2}(t, 1,1) \neq 1 ; \text { at }(p, q)=(1,1)
$$

$\left(H_{3}\right)$ For all $t \in \mathbf{I}$ such that

$$
0 \leq p \leq p_{1}, \quad 0 \leq q \leq q_{1} \Rightarrow \mathcal{H}_{1}(t, p, q) \leq \mathcal{H}_{1}\left(t, p_{1}, q_{1}\right), \quad \mathcal{H}_{2}(t, p, q) \leq \mathcal{H}_{1}\left(t, p_{1}, q_{1}\right) ;
$$

$\left(H_{4}\right)$ For $p, q \geq 0$, there exist real numbers $0<\lambda, \mu<1$, such that for each $t \in \mathbf{I}, \tau \in(0,1)$, we have

$$
\mathcal{H}_{1}(t, \tau p, \tau q) \geq \tau^{\lambda} \mathcal{H}_{1}(t, p, q), \quad \mathcal{H}_{2}(t, \tau p, \tau q) \geq \tau^{\mu} \mathcal{H}_{2}(t, p, q)
$$

Theorem 3.4. Under the assumptions $\left(H_{1}\right)-\left(H_{4}\right)$, the BVP (1) has a unique solution in $\mathbf{C}_{f}$ where $f(t)=\left(t^{\theta_{1}-1}, t^{\theta_{2}-1}\right)$.

Proof. Let $\max \{\lambda, \mu\}=\kappa$ and $(p, q) \in \mathbf{C}$. For each $t \in \mathbf{I}$, using $\left(H_{4}\right)$, we have

$$
\begin{array}{r}
\mathcal{S}_{1}(\tau p, \tau q)(t)=\int_{0}^{1} \mathbf{G}_{1}(t, s) \mathcal{H}_{1}(s, \tau p(s), \tau q(s)) d s \\
\geq \tau^{\lambda} \int_{0}^{1} \mathbf{G}_{1}(t, s) \mathcal{H}_{1}(s, p(s), q(s)) d s=\tau^{\lambda} \mathcal{S}_{1}(p, q)(t) \geq \tau^{\kappa} \mathcal{S}_{1}(p, q)(t),
\end{array}
$$

Analogously, we also get

$$
\mathcal{S}_{2}(\tau p, \tau q)(t) \geq \tau^{\kappa} \mathcal{S}_{2}(p, q)(t)
$$

In view of partial order $\succeq$ on $\mathbf{E} \times \mathbf{E}$ induced by the cone $\mathbf{C}$, we get $\mathcal{S}(\tau p, \tau q) \succeq \tau^{\kappa} \mathcal{S}(p(t), q$ $(t)), \quad \tau \in(0,1),(p, q) \in \mathrm{C}$. Which yields that $\mathcal{S}$ is $\tau$ - concave and nondecreasing operator with respect to the partial order by using hypothesis $\left(H_{4}\right)$. Hence, taking $f \in \mathbf{C}$ for each $t \in \mathbf{I}$ defined by

$$
f(t)=\left(t^{\theta_{1}-1}, t^{\theta_{2}-1}\right)=\left(f_{1}(t), f_{2}(t)\right)
$$

Suppose that 


$$
\mathbf{w}_{1}=\max \left\{\frac{1}{\Gamma\left(\theta_{1}\right)} \int_{0}^{1} \mathcal{H}_{1}(s, 1,1) d s, \frac{1}{\Gamma\left(\theta_{2}\right)} \int_{0}^{1} \mathcal{H}_{2}(s, 1,1) d s\right\}
$$

and

$$
\mathbf{w}_{2}=\max \left\{\frac{1}{\Gamma\left(\theta_{1}\right)} \int_{0}^{1} \mathbf{L}(s) \mathcal{H}_{1}(s, 0,0) d s, \frac{1}{\Gamma\left(\theta_{2}\right)} \int_{0}^{1} \mathbf{K}(s) \mathcal{H}_{2}(s, 0,0) d s\right\} .
$$

Also, from Green's functions, we can obtain that

$$
\mathbf{L}(s)=(1-s)^{\theta_{1}-1}\left(\frac{1+\lambda_{1}}{\lambda_{1}}\right), \mathbf{K}(s)=(1-s)^{\theta_{2}-1}\left(\frac{1+\lambda_{2}}{\lambda_{2}}\right) .
$$

Due to nondecreasing property of $\mathcal{H}_{1}, \mathcal{H}_{2}$ in view of $\left(H_{3}\right)$, we get $\mu>0, v>0$. Therefore, applying (19) together with $\left(H_{4}\right)$, one has

$$
\begin{array}{r}
\mathcal{S}_{1} h(t)=\int_{0}^{1} \mathbf{G}_{1}(t, s) \mathcal{H}_{1}\left(s, f_{1}(s), f_{2}(s)\right) d s \\
=\int_{0}^{1} \mathbf{G}_{1}(t, s) \mathcal{H}_{1}\left(s, s^{\theta_{1}-1}, s^{\theta_{2}-1}\right) d s \leq \int_{0}^{1} \mathbf{G}_{1}(t, s) \mathcal{H}_{1}(s, 1,1) d s \\
\leq\left(\frac{1}{\Gamma\left(\theta_{1}\right)} \int_{0}^{1}(1-s)^{\theta_{1}-1} \mathcal{H}_{1}(s, 1,1) d s\right) t^{\theta_{1}-1} \leq \mu f_{1}(t) .
\end{array}
$$

Similarly, we can get

$$
\mathcal{S}_{2} f(t) \leq \mu f_{2}(t)
$$

Then, we obtain

$$
\mathcal{S} f \preceq \mu f .
$$

Like the aforesaid process, applying Eq. (19) together with $\left(H_{4}\right)$, for each $t \in \mathbf{I}$, one has

$$
\begin{array}{r}
\mathcal{S}_{1} f(t)=\int_{0}^{1} \mathbf{G}_{1}(t, s) \mathcal{H}_{1}\left(s, s^{\theta_{1}-1}, s^{\theta_{2}-1}\right) d s \geq \int_{0}^{1} \mathbf{G}_{1}(t, s) \mathcal{H}_{1}(s, 0,0) d s \\
\geq\left(\frac{1}{\Gamma \theta_{1}} \int_{0}^{1} \mathbf{L}(s) \mathcal{H}_{1}(s, 0,0) d s\right) t^{\theta_{1}-1} \geq v h_{1}(t),
\end{array}
$$

With same fashion, we can obtain 


$$
\mathcal{S}_{2} f(t) \geq v f_{2}(t)
$$

Thus, we have

$$
\mathcal{S} f(t) \succeq v f .
$$

From Eqs. (20) and (21), we produce

$$
v f \preceq \mathcal{S} f \preceq \mu f,
$$

which implies that $\mathcal{S} f \in \mathrm{C}_{f}$. So, thanks to Lemma 2.9, we see that the operator $\mathcal{S}$ is concave; hence, it has at most one fixed point $(p, q) \in \mathbf{C}_{f}$ which is the corresponding solution of BVPs (1). Now, we define the following:

$\left(C_{1}\right) \mathcal{H}_{j}(j=1,2): \mathbf{I} \times \mathbf{R}^{+} \cup\{0\} \times \mathbf{R}^{+} \cup\{0\} \rightarrow \mathbf{R}^{+} \cup\{0\}$ is uniformly bounded and continuous on $\mathbf{I}$ with respect to $t$.

$\left(C_{2}\right)$ Green's functions $\mathbf{G}_{1}(1, s), \mathbf{G}_{2}(1, s)$ satisfy

$$
0<\int_{0}^{1} \mathbf{G}_{1}(1, s) d s<\infty, \quad 0<\int_{0}^{1} \mathbf{G}_{2}(1, s) d s<\infty ;
$$

$\left(C_{3}\right)$ Let these limits hold:

$$
\begin{aligned}
\mathcal{H}_{1}^{\varrho} & =\lim _{p+q \rightarrow \mathrm{e}} \max _{t \in \mathbf{I}} \frac{\mathcal{H}_{1}(t, p, q)}{p+q}, \mathcal{H}_{2}^{\varrho}=\lim _{p+g \rightarrow \varrho} \max _{t \in \mathbf{I}} \frac{\mathcal{H}_{2}(t, p, q)}{p+q}, \\
\mathcal{H}_{1, \mathrm{e}} & =\lim _{p+q \rightarrow \mathrm{e}} \inf _{t \in \mathbf{I}} \frac{\mathcal{H}_{1}(t, p, q)}{p+q}, \mathcal{H}_{2, \mathrm{e}}=\lim _{p+q \rightarrow \mathrm{e} \inf \mathbf{I}} \frac{\mathcal{H}_{2}(t, p, q)}{p+q}, \text { where } \mathrm{\varrho} \in\{0, \infty\} . \\
\delta_{1} & =\max _{t \in \mathbf{I}} \int_{0}^{1} \mathbf{G}_{1}(t, s) d s, \quad \delta_{2}=\max _{t \in \mathbf{I}} \int_{0}^{1} \mathbf{G}_{2}(t, s) d s .
\end{aligned}
$$

Theorem 3.5. Assume that the conditions $\left(C_{1}\right)-\left(C_{3}\right)$ together with given assumptions are satisfied:

$\left(H_{5}\right) \mathcal{H}_{1,0}\left(\gamma_{1}^{2} \int_{\theta}^{1-\theta} \mathbf{G}_{1}(1, s) d s\right)>1, \mathcal{H}_{1, \infty}\left(\gamma_{1}^{2} \int_{\theta}^{1-\theta} \mathbf{G}_{1}(1, s) d s\right)>1$ and

$$
\mathcal{H}_{2,0}\left(\gamma_{2}^{2} \int_{\theta}^{1-\theta} \mathbf{G}_{2}(1, s) d s\right)>1, \mathcal{H}_{2, \infty}\left(\gamma_{2}^{2} \int_{\theta}^{1-\theta} \mathbf{G}_{2}(1, s) d s\right)>1 .
$$

Moreover, $\mathcal{H}_{1,0}=\mathcal{H}_{2,0}=\mathcal{H}_{1, \infty}=\mathcal{H}_{2, \infty}=\infty$ also hold:

$\left(H_{6}\right)$ There exists constant $\alpha>0$ such that 


$$
\max _{t \in \mathbf{I},(p, q) \in \partial \mathbf{C}_{\alpha}} \mathcal{H}_{1}(t, p, q)<\frac{\alpha}{2 \delta_{1}}
$$

and

$$
\max _{t \in \mathbf{I},(p, q) \in \partial \mathbf{C}_{\alpha}} \mathcal{H}_{2}(t, p, q)<\frac{\alpha}{2 \delta_{2}}
$$

Then, the system (1) of BVPs has at least two positive solutions $(p, q),(\bar{p}, \bar{q})$ which obeying

$$
0<\|(p, q)\|_{\mathbf{E} \times \mathbf{E}}<\alpha<\|(\bar{p}, \bar{q})\|_{\mathbf{E} \times \mathbf{E}} .
$$

Proof. Assume that $\left(H_{5}\right)$ holds, and consider $\epsilon, \alpha, \lambda$ such that $0<\epsilon<\alpha<\lambda$. Further we define a set by

$$
\Omega_{r}=\left\{(u, v) \in \mathbf{E} \times \mathbf{E}:\|(u, v)\|_{\mathbf{E} \times \mathbf{E}}<r\right\}, \text { where } r \in\{\boldsymbol{\epsilon}, \alpha, \lambda\} .
$$

Now, if

$$
\mathcal{H}_{1,0}\left(\gamma_{1}^{2} \int_{\theta}^{1-\theta} \mathbf{G}_{1}(1, s) d s\right)>1 \text { and } \mathcal{H}_{2,0}\left(\gamma_{2}^{2} \int_{\theta}^{1-\theta} \mathbf{G}_{2}(1, s) d s\right)>1
$$

Then, obviously, we can obtain that

$$
\|\mathcal{S}(p, q)\|_{\mathbf{E} \times \mathbf{E}} \geq\|(p, q)\|_{\mathbf{E} \times \mathbf{E}}, \text { for }(p, q) \in \mathbf{C} \cap \partial \Omega_{\varepsilon} .
$$

Now, if $\mathcal{H}_{1, \infty}\left(\gamma_{1}^{2} \int_{\theta}^{1-\theta} \mathbf{G}_{1}(1, s) d s\right)>1$ and $\mathcal{H}_{2, \infty}\left(\gamma_{2}^{2} \int_{\theta}^{1-\theta} \mathbf{G}_{2}(1, s) d s\right)>1$.

Then, like the proof of Eq. (23), we have

$$
\|\mathcal{S}(p, q)\|_{\mathbf{E} \times \mathbf{E}} \geq\|(p, q)\|_{\mathbf{E} \times \mathbf{E}}, \text { for }(p, q) \in \mathbf{C} \cap \partial \Omega_{\lambda} .
$$

Also, from $\left(H_{5}\right)$ and $(p, q) \in \mathbf{C} \cap \partial \Omega_{\alpha}$, we get

$$
\begin{aligned}
& \left|\mathcal{S}_{1}(p, q)(t)\right|=\left|\int_{0}^{1} \mathbf{G}_{1}(t, s) \mathcal{H}(s, u(s), v(s)) d s\right| \\
& \quad \leq \int_{0}^{1} \mathbf{G}_{1}(1, s)\left|\mathcal{H}_{1}(s, p(s), q(s))\right| d s .
\end{aligned}
$$

From which we have

$$
\left\|\mathcal{S}_{1}(p, q)\right\|_{\mathrm{E} \times \mathbf{E}}<\frac{\alpha}{2 \varrho_{1}} \int_{0}^{1} \mathbf{G}_{1}(1, s) d s=\frac{\alpha}{2} .
$$

Similarly, we have $\left\|\mathcal{S}_{1}(p, q)\right\|_{\mathrm{E} \times \mathbf{E}}<\frac{\alpha}{2}$ as $(p, q) \in \mathbf{C} \cap \partial \Omega_{\alpha}$. Hence, we have 


$$
\|\mathcal{S}(p, q)\|_{\mathbf{E} \times \mathbf{E}}<\|(p, q)\|_{\mathbf{E} \times \mathbf{E}}, \text { for }(p, q) \in \mathbf{C} \cap \partial \Omega_{\alpha} .
$$

Now, applying Lemma 2.11 to Eqs. (23) and (25) yields that $\mathcal{S}$ has a fixed point $(p, q) \in \mathbf{C} \cap\left(\bar{\Omega}_{\alpha} \backslash \mathbf{C}_{\varepsilon}\right)$ and a fixed point in $(\bar{p}, \bar{q}) \in \mathbf{C} \cap\left(\bar{\Omega}_{\lambda} \backslash \Omega_{\alpha}\right)$. Hence, we conclude that the system of BVPs (1) has at least two positive solutions $(p, q),(\bar{p}, \bar{q})$ such that $\|(p, q)\|_{\mathbf{E} \times \mathbf{E}} \neq \alpha$ and $\|(\bar{p}, \bar{q})\|_{\mathbf{E} \times \mathbf{E}} \neq \alpha$. Thus, relation (22) holds.

Theorem 3.6. Consider that $\left(C_{1}\right)-\left(C_{3}\right)$ together with the following hypothesis are satisfied:

$\left(H_{7}\right) \delta_{1} \mathcal{H}_{1,0}<1, \delta_{1} \mathcal{H}_{1, \infty}<1 ; \quad \delta_{2} \mathcal{H}_{1,0}<1$, and $\delta_{2} \mathcal{H}_{2, \infty}<1$;

$\left(H_{8}\right)$ There exist $\rho>0$ such that

$$
\begin{aligned}
\max _{t \in \mathbf{I},(p, q) \in \partial \mathbf{C}_{\alpha}} \gamma_{1}^{2} \mathcal{H}_{1}(t, p, q) & >\frac{\alpha}{2}\left(\int_{\theta}^{1-\theta} \mathbf{G}_{1}(1, s) d s\right)^{-1}, \\
\max _{t \in \mathbf{I},(p, q) \in \partial \mathbf{C}_{\alpha}} \gamma_{2}^{2} \mathcal{H}_{2}(t, p, q) & >\frac{\alpha}{2}\left(\int_{\theta}^{1-\theta} \mathrm{G}_{2}(1, s) d s\right)^{-1}
\end{aligned}
$$

such that

$$
0<\|(p, q)\|_{\mathbf{E} \times \mathbf{E}}<\alpha<\|(\bar{p}, \bar{q})\|_{\mathbf{E} \times \mathbf{E}} .
$$

Then, the proposed coupled system of BVPs (1) has at least two positive solutions.

Proof. Proof is like the proof of Theorem 3.4.

Analogously, we deduce from Theorem 3.5 and 3.6 the following results for multiplicity of solutions to the system (1) of BVPs.

Theorem 3.7. Under the conditions $\left(C_{1}\right)-\left(C_{3}\right)$, there exist $2 k$ positive numbers $\mathbf{a}_{j}, \widehat{\mathbf{a}}_{j}, j=1,2 \ldots k$ with $\quad \mathbf{a}_{1}<\gamma_{1} \widehat{\mathbf{a}}_{1}<\widehat{\mathbf{a}}_{1}<\mathbf{a}_{2}<\gamma_{1} \widehat{\mathbf{a}}_{2}<\widehat{\mathbf{a}}_{2} \ldots \mathbf{a}_{k}<\gamma_{1} \widehat{\mathbf{a}}_{k}<\widehat{\mathbf{a}}_{k}$ and $\mathbf{a}_{1}<\gamma_{2} \widehat{\mathbf{a}}_{1}<\widehat{\mathbf{a}}_{1}<\mathbf{a}_{2}<\gamma_{2} \widehat{\mathbf{a}}_{2}<$ $\widehat{\mathbf{a}}_{2} \ldots \mathbf{a}_{k}<\gamma_{2} \widehat{\mathbf{a}}_{k}<\widehat{\mathbf{a}}_{k}$ such that.

$\left(H_{9}\right) \mathcal{H}_{1}(t, p(t), q(t))\left(\gamma_{1} \int_{0}^{1} \mathbf{G}_{1}(1, s) d s\right) \geq \mathbf{a}_{j}$, for $(t, p, q) \in \mathbf{I} \times\left[\gamma_{1} \mathbf{a}_{j}, \mathbf{a}_{j}\right] \times\left[\gamma_{2} \mathbf{a}_{j}, \mathbf{a}_{j}\right]$, and

$$
\mathcal{H}_{1}(t, p(t), q(t)) \delta_{1} \leq \widehat{\mathbf{a}}_{i}, \text { for }(t, p, q) \in \mathbf{I} \times\left[\gamma_{1} \widehat{\mathbf{a}}_{j}, \widehat{\mathbf{a}}_{j}\right] \times\left[\gamma_{2} \mathbf{a}_{j}, \mathbf{a}_{i}\right], j=1,2 \ldots k ;
$$

$\left(H_{10}\right) \mathcal{H}_{2}(t, p(t), q(t))\left(\gamma_{2} \int_{0}^{1} \mathbf{G}_{2}(1, s) d s\right) \geq \mathbf{a}_{j}$, for $(t, p, q) \in \mathbf{I} \times\left[\gamma_{1} \mathbf{a}_{j}, \mathbf{a}_{j}\right] \times\left[\gamma_{2} \mathbf{a}_{j}, \mathbf{a}_{j}\right]$, and 


$$
\mathcal{H}_{1}(t, p(t), q(t)) \delta_{2} \leq \widehat{\mathbf{a}}_{j}, \text { for }(t, p, q) \in \mathbf{I} \times\left[\gamma_{1} \mathbf{a}_{j}, \mathbf{a}_{j}\right] \times\left[\gamma_{2} \widehat{\mathbf{a}}_{j}, \widehat{\mathbf{a}}_{j}\right], j=1,2 \ldots k
$$

Then, system (1) of BVPs has at least $k$ solutions $\left(p_{j}, q_{j}\right)$, satisfying

$$
\mathbf{a}_{j} \leq\left\|\left(p_{j}, q_{j}\right)\right\|_{\mathbf{E} \times \mathbf{E}} \leq \widehat{\mathbf{a}}_{j}, j=1,2 \ldots k .
$$

Further, if assumptions $\left(C_{1}\right)-\left(C_{3}\right)$ hold such that there exist $2 k$ positive numbers $\mathbf{b}_{j}, \widehat{\mathbf{b}}_{j}, j=1,2 \ldots k$, with

$$
\mathbf{b}_{1}<\widehat{\mathbf{b}}_{1}<\mathbf{b}_{2}<\widehat{\mathbf{b}}_{2} \ldots<\mathbf{b}_{k}<\widehat{\mathbf{b}}_{k}
$$

together with following hypothesis hold:

$\left(H_{11}\right) \mathcal{H}_{1}(t, p, q)$ and $\mathcal{H}_{2}(t, p, q)$ are nondecreasing on $\left[0, \widehat{\mathbf{b}}_{k}\right]$ for all $t \in \mathbf{I}$;

$$
\begin{gathered}
\left(H_{11}\right) \mathcal{H}_{1}(t, p(t), q(t))\left(\gamma_{1} \int_{\theta}^{1-\theta} \mathbf{G}_{1}(1, s) d s\right) \geq \mathbf{b}_{j}, \mathcal{H}_{1}(t, p(t), q(t)) \delta_{1} \leq \widehat{\mathbf{b}}_{j}, j=1,2 \ldots k, \\
\mathcal{H}_{2}(t, u(t), v(t))\left(\gamma_{2} \int_{\theta}^{1-\theta} \mathbf{G}_{2}(1, s) d s\right) \geq \mathbf{b}_{j}, \mathcal{H}_{2}(t, p(t), q(t)) \delta_{2} \leq \widehat{\mathbf{b}}_{j}, j=1,2 \ldots k .
\end{gathered}
$$

Then, system (1) of BVPs has at least $k$ solutions $\left(p_{j}, q_{j}\right)$, satisfying

$$
\mathbf{b}_{j} \leq\left\|\left(p_{j}, q_{j}\right)\right\|_{\mathrm{E} \times \mathbf{E}} \leq \widehat{\mathbf{b}}_{j}, \quad j=1,2 \ldots k .
$$

\section{Hyers-Ulam stability}

Definition 4.1. ([31, Definition 2]) Consider a Banach space $\mathbf{E} \times \mathbf{E}$ such that $\mathcal{S}_{1}, \mathcal{S}_{2}: \mathbf{E} \times \mathbf{E} \rightarrow \mathbf{E} \times \mathbf{E}$ be the two operators. Then, the operator system provided by

$$
\left\{\begin{array}{l}
p(t)=\mathcal{S}_{1}(p, q)(t), \\
q(t)=\mathcal{S}_{2}(p, q)(t)
\end{array}\right.
$$

is called Hyers-Ulam stability if we can find $\mathscr{C}_{i}(i=1,2,3,4)>0$, such that for each $\rho_{i}(i=1,2)>0$ and for each solution $\left(p^{*}, q^{*}\right) \in \mathbf{E} \times \mathbf{E}$ of the inequalities given by

$$
\left\{\begin{array}{l}
\left\|p^{*}-\mathcal{H}_{1}\left(p^{*}, q^{*}\right)\right\|_{\mathrm{E} \times \mathbf{E}} \leq \rho_{1}, \\
\left\|q^{*}-\mathcal{H}_{2}\left(p^{*}, q^{*}\right)\right\|_{\mathrm{E} \times \mathbf{E}} \leq \rho_{2},
\end{array}\right.
$$


there exist a solution $(\bar{p}, \bar{q}) \in \mathbf{E} \times \mathbf{E}$ of system (26) which satisfy

$$
\left\{\begin{array}{l}
\left\|p^{*}-\bar{p}\right\|_{\mathrm{E} \times \mathrm{E}} \leq \mathcal{C}_{1} \rho_{1}+\mathcal{C}_{2} \rho_{2} \\
\left\|q^{*}-\bar{q}\right\|_{\mathrm{E} \times \mathrm{E}} \leq \mathcal{C}_{3} \rho_{1}+\mathcal{C}_{4} \rho_{2} .
\end{array}\right.
$$

Definition 4.2. If $\lambda_{i}$, for $i=1,2, \cdots, n$ be the (real or complex) eigenvalues of a matrix $\mathbf{M} \in \mathcal{C}^{n \times n}$, then the spectral radius $\rho(\mathbf{M})$ is defined by

$$
\rho(\mathbf{M})=\max \left\{\left|\lambda_{i}\right|, \text { for } i=1,2, \cdots, n\right\} .
$$

Further, the matrix will converge to zero if $\rho(\mathbf{M})<1$..

Theorem 4.3. ([31, Theorem 4]) Consider a Banach space $\mathbf{E} \times \mathbf{E}$ with $\mathcal{S}_{1}, \mathcal{S}_{2}: \mathbf{E} \times \mathbf{E} \rightarrow \mathbf{E} \times \mathbf{E}$ be the two operators such that

$$
\left\{\begin{array}{l}
\left\|\mathcal{S}_{1}(p, q)-\mathcal{S}_{1}\left(p^{*}, q^{*}\right)\right\|_{\mathbf{E} \times \mathbf{E}} \leq \mathcal{C}_{1}\left\|p-p^{*}\right\|_{\mathbf{E} \times \mathbf{E}}+\mathcal{C}_{2}\left\|q-q^{*}\right\|_{\mathbf{E} \times \mathbf{E}} \\
\left\|\mathcal{S}_{2}(p, q)-\mathcal{S}_{2}\left(p^{*}, q^{*}\right)\right\|_{\mathbf{E} \times \mathbf{E}} \leq \mathcal{C}_{3}\left\|p-p^{*}\right\|_{\mathbf{E} \times \mathbf{E}}+\mathcal{C}_{4}\left\|q-q^{*}\right\|_{\mathbf{E} \times \mathbf{E}} \\
\text { forall }(p, q),\left(p^{*}, q^{*}\right) \in \mathbf{E} \times \mathbf{E},
\end{array}\right.
$$

and if the matrix

$$
\mathbf{M}=\left[\begin{array}{ll}
\mathcal{C}_{1} & \mathcal{C}_{2} \\
\mathcal{C}_{3} & \mathcal{C}_{4}
\end{array}\right]
$$

converges to zero ([31, Theorem 1]), then the fixed points corresponding to operatorial system (26) are Hyers-Ulam stable.

For the stability results, the following should be hold:

$\left(H_{13}\right)$ Under the continuity of $\mathcal{H}_{i}, i=1,2$, there exist $a_{i}, b_{i} \in c(0,1), \quad i=1,2$ and $(p, q),(\bar{p}, \bar{q})$ such that

$$
\left|\mathcal{H}_{i}(t, p, q)-\mathcal{H}_{i}(t, \bar{p}, \bar{q})\right| \leq a_{i}(t)|p-\bar{p}|+b_{i}(t)|q-\bar{q}|, \quad i=1,2
$$

In this section, we study Hyers-Ulam stability for the solutions of our proposed system. Thanks to Definition 4.1 and Theorem 4.3 , the respective results are received.

Theorem 4.4. Suppose that the assumptions $\left(H_{13}\right)$ along with condition that matrix

$$
\mathbf{M}=\left[\begin{array}{ll}
\int_{0}^{1} \mathbf{G}_{1}(1, s) a_{1}(s) d s & \int_{0}^{1} \mathbf{G}_{1}(1, s) b_{1}(s) d s \\
\int_{0}^{1} \mathbf{G}_{2}(1, s) a_{2}(s) d s & \int_{0}^{1} \mathbf{G}_{2}(1, s) b_{2}(s) d s
\end{array}\right]
$$

is converging to zero. Then, the solutions of (1) are Hyers-Ulam stable.

Proof. In view of Theorem 4.3, we have 


$$
\begin{aligned}
& \left\|\mathcal{S}_{1}(p, q)-\mathcal{S}_{1}(\bar{p}, \bar{q})\right\|_{\mathbf{E} \times \mathbf{E}} \leq \int_{0}^{1} \mathbf{G}_{1}(1, s) a_{1}(s)\|p-\bar{p}\|_{\mathbf{E} \times \mathbf{E}} d s+\int_{0}^{1} \mathbf{G}_{1}(1, s) b_{1}(s)\|q-\bar{q}\|_{\mathbf{E} \times \mathbf{E}} d s \\
& \left\|\mathcal{S}_{2}(p, q)-\mathcal{S}_{2}(\bar{p}, \bar{q})\right\|_{\mathbf{E} \times \mathbf{E}} \leq \int_{0}^{1} \mathbf{G}_{2}(1, s) a_{2}(s)\|p-\bar{p}\|_{\mathbf{E} \times \mathbf{E}} d s+\int_{0}^{1} \mathbf{G}_{2}(1, s) b_{2}(s)\|q-\bar{q}\|_{\mathbf{E} \times \mathbf{E}} d s .
\end{aligned}
$$

From which we get

$$
\begin{aligned}
& \left.\left\|\mathcal{S}_{1}(p, q)-\mathcal{S}_{1}(\bar{p}, \bar{q})\right\|_{\mathbf{E} \times \mathbf{E}} \leq\left[\int_{0}^{1} \mathbf{G}_{1}(1, s) a_{1}(s) d s\right]\right]\|p-\bar{p}\|_{\mathbf{E} \times \mathbf{E}}+\left[\int_{0}^{1} \mathbf{G}_{1}(1, s) b_{1}(s) d s\right]\|q-\bar{q}\|_{\mathbf{E} \times \mathbf{E}} \\
& \left\|\mathcal{S}_{2}(p, q)-\mathcal{S}_{2}(\bar{p}, \bar{q})\right\|_{\mathbf{E} \times \mathbf{E}} \leq\left[\int_{0}^{1} \mathbf{G}_{2}(1, s) a_{2}(s) d s\right]\|p-\bar{p}\|_{\mathbf{E} \times \mathbf{E}}+\left[\int_{0}^{1} \mathbf{G}_{2}(1, s) b_{2}(s) d s\right]\|q-\bar{q}\|_{\mathbf{E} \times \mathbf{E}} .
\end{aligned}
$$

Hence, we get

$$
\|\mathcal{S}(p, q)-\mathcal{S}(\bar{p}, \bar{q})\|_{\mathbf{E} \times \mathbf{E}} \leq \mathbf{M}\|(p, q)-(\bar{p}, \bar{q})\|_{\mathbf{E} \times \mathbf{E}}
$$

where $\mathbf{M}=\left[\begin{array}{ll}\int_{0}^{1} \mathbf{G}_{1}(1, s) a_{1}(s) d s & \int_{0}^{1} \mathbf{G}_{1}(1, s) b_{1}(s) d s \\ \int_{0}^{1} \mathbf{G}_{2}(1, s) a_{2}(s) d s & \int_{0}^{1} \mathbf{G}_{2}(1, s) b_{2}(s) d s\end{array}\right]$. Hence, we received the required results.

\section{Illustrative examples}

Example 5.1. Consider the given system of BVPS

$$
\left\{\begin{array}{l}
\mathscr{D}^{\frac{7}{2}} p(t)+\left(1-t^{2}\right)+[p(t) q(t)]^{\frac{1}{3}}=0, \quad \mathscr{D}^{\frac{11}{3}} q(t)+1+t+[p(t) q(t)]^{\frac{1}{4}}=0, \quad t \in(0,1), \\
p(t)=p^{\prime}(t)=p^{\prime \prime}(t)=q(t)=q^{\prime}(t)=q^{\prime \prime}(t)=0, \text { at } t=0, \\
p(1)=p\left(\frac{1}{4}\right), q(1)=q\left(\frac{1}{3}\right) .
\end{array}\right.
$$

Clearly, $\mathcal{H}_{1}(t, p, q) \neq 0, \mathcal{H}_{2}(t, p, q) \neq 0$, at $(p, q)=(0,0)$, and $\mathcal{H}_{1}(t, p, q) \neq 0, \mathcal{H}_{2}(t, p, q) \neq 0$, at $(p, q)=(1,1)$. Simple computation yields that $\mathcal{H}_{1}, \mathcal{H}_{2}$ are nondecreasing for every $t \in(0,1)$. Also, for $\tau, t \in(0,1)$, and $p, q \geq 0$, one has $\max \left\{\frac{1}{4}, \frac{1}{3}\right\}=\frac{1}{3}$,

$$
\mathcal{H}_{1}(t, \tau p, \tau q) \geq \tau^{\frac{1}{3}} \mathcal{H}_{1}(t, p, q), \quad \mathcal{H}_{2}(t, \tau p, \tau q) \geq \tau^{\frac{1}{3}} \mathcal{H}_{2}(t, p, q)
$$

Thus, all the conditions of Theorem 3.4 are fulfilled, so the system (32) of BVPs has unique positive solution in $\mathbf{B}_{f}$ where $f(t)=\left(t^{\frac{5}{2}}, t^{\frac{9}{2}}\right)$.

Example 5.2. Consider the following system of BVPs:

$$
\left\{\begin{array}{l}
\mathscr{D}^{\frac{9}{2}} p(t)+(1+t)^{2}+[p(t)+q(t)]^{3}=0, \quad \mathscr{D}^{\frac{9}{2}} q(t)+1+t+[p(t)+q(t)]^{2}=0, \quad t \in(0,1), \\
p^{(j)}(t)=q^{(j)}(t)=0, \quad j=0,1,2,3, \text { at } t=0, \\
p(1)=p\left(\frac{1}{2}\right), q(1)=q\left(\frac{1}{2}\right) .
\end{array}\right.
$$


It is obvious that $\mathcal{H}_{1}(t, p, q) \neq 0, \quad \mathcal{H}_{2}(t, p, q) \neq 0, \quad$ at $(p, q)=(0,0)$, and $\mathcal{H}_{1}(t, p, q) \neq 0$, $\mathcal{H}_{2}(t, p, q) \neq 0$, at $(p, q)=(1,1)$. Also, an easy computation yields that $\mathcal{H}_{1}, \mathcal{H}_{2}$ are nondecreasing for each $t \in(0,1)$. Moreover, for $\tau, t \in(0,1)$, and $p, q \geq 0$, we see that $\max \{3,2\}=3$,

$$
\mathcal{H}_{1}(t, \tau p, \tau q) \geq \tau^{3} \mathcal{H}_{1}(t, p, q), \quad \mathcal{H}_{2}(t, \tau p, \tau q) \geq \tau^{3} \mathcal{H}_{2}(t, p, q) .
$$

Thus, all the assumption of Theorem 3.4 is fulfilled, so the coupled system (33) has a unique positive solution in $\mathbf{B}_{f}$ where $f(t)=\left(t^{\frac{3}{4}}, t^{\frac{4}{3}}\right)$.

Example 5.3. Consider the following system of BVPs:

$$
\left\{\begin{array}{l}
\mathscr{D}^{\frac{7}{2}} p(t)=\frac{t}{40}+\frac{t}{20} \cos |p(t)|+\frac{t^{2}}{20} \sin |q(t)|, \quad t \in(0,1), \\
\mathscr{D}^{\frac{7}{2}} q(t)=\frac{t^{2}}{50}+\frac{t^{2}}{60} \sin |p(t)|+\frac{t}{60} \cos |q(t)|, \quad t \in(0,1), \\
p^{(j)}(t)=q^{(j)}(t)=0, \quad j=0,1,2, \text { at } t=0, \\
p(1)=p\left(\frac{1}{2}\right), q(1)=q\left(\frac{1}{2}\right) .
\end{array}\right.
$$

From system (33), we see that

$$
\left|\mathcal{H}_{1}(t, p, q)\right| \leq \frac{t}{40}+\frac{t}{20} \cos |p(t)|+\frac{t^{2}}{20} \sin |q(t)|
$$

and

$$
\left|\mathcal{H}_{2}(t, p, q)\right| \leq \frac{t^{2}}{50}+\frac{t^{2}}{60} \sin |p(t)|+\frac{t}{60} \cos |q(t)|
$$

where $\varphi_{1}(t)=\frac{t}{40}, \quad \varphi_{2}(t)=\frac{t^{2}}{50}, \quad \psi_{1}(t)=\frac{t}{20}, \quad \psi_{2}(t)=\frac{t^{2}}{60}, \quad \sigma_{1}(t)=\frac{t^{2}}{20}, \quad \sigma_{2}(t)=\frac{t}{60} . \quad$ Also, $\eta=\xi=\frac{1}{2}, \lambda_{1}=\lambda_{2}=0.17677$. Thus, by computation, we have

$$
\mathbf{G}_{j}(1, s)=6.65710 \frac{(1-s)^{\frac{5}{2}}}{\Gamma\left(\frac{7}{2}\right)}, \text { for } j=1,2 .
$$

Upon computation, we get

$$
\Delta_{1}=\int_{0}^{1} \mathbf{G}_{1}(1, s) \varphi_{1}(s) d s=0.003577<\infty, \quad \Delta_{2}=\int_{0}^{1} \mathbf{G}_{2}(1, s) \varphi_{2}(s) d s=0.000924<\infty .
$$

Similarly, we can also compute.

$\Lambda_{1}=\int_{0}^{1} \mathbf{G}_{1}(1, s)\left[\psi_{1}(s)+\sigma_{1}(s)\right] d s=0.03092853<1, \quad \Lambda_{2}=\int_{0}^{1} \mathbf{G}_{2}(1, s)\left[\psi_{2}(s)+\sigma_{2}(s)\right] d s=0.00289<1$. Further, we see that $\max \{0.007626,0.00185\}=0.007626$. So, all the conditions of Theorem 3.3 are satisfied. So, the BVP (34) has at least one solution and the solution lies in

$$
\mathcal{E}=\left\{(p, q) \in \mathbf{C}:\|(p, q)\|_{\mathrm{E} \times \mathbf{E}}<0.007626\right\} .
$$


Example 5.4. Taking the following system of BVPS

$$
\left\{\begin{array}{l}
\mathscr{D}^{\frac{11}{2}} p(t)+\frac{[p(t)+q(t)]^{2}+1}{\left(15+t^{2}\right) \delta_{1}}=0, \quad \mathscr{D}^{\frac{11}{2}} q(t)+\frac{[p(t)+q(t)]^{2}+t}{\left(15+t^{2}\right) \delta_{2}}=0, \quad t \in(0,1), \\
p^{(j)}(t)=q^{(j)}(t)=0, \quad j=0,1,2,3,4, \text { at } t=0 \\
p(1)=p\left(\frac{1}{4}\right), \quad q(1)=q\left(\frac{1}{4}\right) .
\end{array}\right.
$$

It is simple to check that $\mathcal{H}_{1,0}=\mathcal{H}_{2,0}=\mathcal{H}_{1, \infty}=\mathcal{H}_{2, \infty}=\infty$. Also, for any $(t, p, q) \in \mathbf{I} \times \mathbf{I} \times \mathbf{I}$, we see that

$$
\begin{aligned}
\mathcal{H}_{1}(t, p, q) & \leq \frac{1}{3 \delta_{1}} \\
\mathcal{H}_{2}(t, p, q) & \leq \frac{1}{3 \delta_{2}} .
\end{aligned}
$$

Thus, all the assumptions of Theorem 3.5 are satisfied with taking $\alpha=1$, so the coupled system (35) has two solutions satisfying $0<\|(p, q)\|_{\mathrm{E} \times \mathbf{E}}<1<\|(p, q)\|_{\mathrm{E} \times \mathbf{E}}$.

Example 5.5. Consider the following coupled systems of boundary value problems:

$$
\left\{\begin{array}{l}
\mathscr{D}^{\frac{5}{2}} p(t)+\Gamma\left(\frac{5}{2}\right)\left[\frac{t p(t)}{16}+\frac{t^{2} q(t)}{32}\right]=0, t \in(0,1) \\
\mathscr{D}^{\frac{5}{2}} q(t)+\Gamma\left(\frac{5}{2}\right)\left[\frac{9 t^{2}|\cos (p(t))|}{16 \sqrt{\pi}}+\frac{9 t|\cos (q(t))|}{32 \sqrt{\pi}}\right]=0, t \in(0,1) \\
p^{(j)}(t)=q^{(j)}(t)=0, \quad j=0,1,2, \text { at } t=0 \\
p(1)=p\left(\frac{1}{2}\right), \quad q(1)=q\left(\frac{1}{2}\right)
\end{array}\right.
$$

Here, $a_{1}(t)=\Gamma\left(\frac{5}{2}\right) \frac{t}{16}, b_{1}(t)=\Gamma\left(\frac{5}{2}\right) \frac{t^{2}}{32}, a_{2}(t)=\Gamma\left(\frac{5}{2}\right) \frac{9 t^{2}}{16 \sqrt{\pi}}, b_{2}(t)=\Gamma\left(\frac{5}{2}\right) \frac{9 t}{32 \sqrt{\pi}}$. Moreover

$$
\mathbf{M}=\left[\begin{array}{ll}
\int_{0}^{1} \mathbf{G}_{1}(1, s) a_{1}(s) d s & \int_{0}^{1} \mathbf{G}_{1}(1, s) b_{1}(s) d s \\
\int_{0}^{1} \mathbf{G}_{2}(1, s) a_{2}(s) d s & \int_{0}^{1} \mathbf{G}_{2}(1, s) b_{2}(s) d s
\end{array}\right]=\left[\begin{array}{ll}
0.0460 & 0.0007 \\
0.0068 & 0.0058
\end{array}\right] .
$$

Here, $\rho(\mathbf{M})=4.61 \times 10^{-2}<1$. Therefore, matrix $\mathbf{M}$ converges to zero, and hence the solutions of (36) are Hyers-Ulam stable by using Theorem 4.4 .

\section{Conclusion}

We have developed a comprehensive theory on existence of solutions and its Hyers-Ulam stability for system of multipoint BVP of FDEs. The concerned theory has been enriched by providing suitable examples. 


\section{Acknowledgements}

We are very thankful to the reviewers for his/her careful reading and suggestion which improved this chapter very well.

\section{Conflict of interest}

We declare the there is no conflict of interest regarding this chapter.

\section{Research funder}

This work has been supported by the National Natural Science Foundation of China (11571378).

\section{Author details}

Kamal Shah ${ }^{1}$ and Yongjin $\mathrm{Li}^{2 *}$

*Address all correspondence to: stslyj@mail.sysu.edu.cn

1 Department of Mathematics, University of Malakand, Dir(L), Khyber Pakhtunkhwa, Pakistan

2 Department of Mathematics, Sun Yat-sen University, Guanghou, China

\section{References}

[1] Hilfer R. Applications of Fractional Calculus in Physics. Singapore: World Scientific; 2000

[2] Kilbas AA, Srivastava H, Trujillo J. Theory and application of fractional differential equations, North Holland Mathematics Studies. Vol. 204. Amsterdam: Elseveir; 2006

[3] Miller KS, Ross B. An Introduction to the Fractional Calculus and Fractional Differential Equations. New York: Wiley; 1993

[4] Podlubny I. Fractional differential equations. New York: Academic press; 1993

[5] Podlubny I. Fractional differential equations. In: Mathematics in Science and Engineering. New York: Academic Press; 1999

[6] Jalili M, Samet B. Existence of positive solutions to a coupled system of fractional differential equation. Mathematical Methods in Applied Science. 2015;38:1014-1031 
[7] Khan RA, Shah K. Existence and uniqueness of solutions to fractional order multi-point boundary value problems. Communications on Pure and Applied Analysis. 2015;19:515-526

[8] Shah K, Khan RA. Study of solution to a toppled system of fractional differential equations with integral boundary. International Journal of Applied and Computational Mathematics. 2016;2(3) 19 pages

[9] Shah K, Khalil H, Khan RA. Investigation of positive solution to a coupled system of impulsive boundary value problems for nonlinear fractional order differential equations. Journal of Chaos, Solitons and Fractals. 2015;77:240-246

[10] Ahmad B, Alsaedi A. Existence and uniqueness of solutions for coupled systems of higher-order nonlinear fractional differential equations. Fixed Point Theory Applications. 2010 Article ID 364560

[11] Su X. Boundary value problem for a coupled system of nonlinear fractional differential equations. Applied Mathematics Letters. 2009;22:64-69

[12] Ahmad B, Nieto JJ. Existence results for a coupled system of nonlinear fractional differential equations with three-point boundary conditions. Computers \& Mathematcs with Applications. 2009;58:18381843

[13] Yang W. Positive solution to nonzero boundary values problem for a coupled system of nonlinear fractional differential equations. Computers \& Mathematcs with Applications. 2012;63:288-297

[14] Bai Z, Lü H. Positive solutions for boundary value problem of nonlinear fractional differential equation. Journal of Mathematical Analysis and Applications. 2005;311:495-505

[15] Kaufmann ER, Mboumi E. Positive solutions for boundary value problem of a nonlinear fractional differential equations, Electronic Journal of Qualitative Theory of Differential Equations. 2008;8:1-11

[16] Shah K, Khan RA. Existence and uniqueness of positive solutions to a coupled system of nonlinear fractional order differential equations with anti periodic boundary conditions. Differential Equations and Applications. 2015;7(2):245-262

[17] Shah K, Khalil H, Khan RA. Upper and lower solutions to a coupled system of nonlinear fractional differential equations. Progress Fractional Differential Applications. 2016;2(1): $1-10$

[18] Zhang SQ. Positive solutions for boundary value problem problems of nonlinear fractional differential equations. Electronic Journal of Differential Equations. 2006;2006:1-12

[19] Wang J, Xiang H, Lu Z. Positive solutions to nonzero boundary value problem for a coupled system of nonlinear fractional differential equations, International Journal of Differential Equations. 2010. 12-pages

[20] Ulam SM. Problems in Modern Mathematics. New York, USA: John Wiley and Sons; 1940

[21] Ulam SM. A Collection of Mathematical Problems. New York: Interscience; 1960 
[22] Obloza M. Hyers stability of the linear differential equation. Rocznik Nauk-Dydakt. Prace Mat. 1993;13:259-270

[23] Wang J, Li X. Ulam-Hyers stability of fractional Langevin equations. Applied Mathematics and Computation. 2015;258:72-83

[24] Stamova I. Mittag-Leffler stability of impulsive differential equations of fractional order. Quarterly of Applied Mathematics. 2015;73(3):525-535

[25] Kumama P, Ali A, Shah K, Khan RA. Existence results and Hyers-Ulam stability to a class of nonlinear arbitrary order differential equations. Journal of Nonlinear Science and Applications. 2017;10:2986-2997

[26] Wang J, Lv L, Zhou Y. Ulam stability and data dependence for fractional differential equations with Caputo derivative. Electronic Journal of Qualitative Theory of Differential Equations. 2011;(63):1-10

[27] Haq F, Shah K, Rahman G, Shahzad M. Hyers-Ulam stability to a class of fractional differential equations with boundary conditions. International Journal of Applied Computational Mathematics. 2017:1-13

[28] Deimling K. Nonlinear Functional Analysis. New York: Springer-Verlag; 1985

[29] Zeidler E. Non Linear Functional Analysis and Its Applications. New York, USA: Springer; 1986

[30] Agarwal R, Meehan M, Regan DO. Fixed Points Theory and Applications. Cambridge: Cambridge University Press; 2004

[31] Urs C. Coupled fixed point theorem and applications to periodic boundary value problem. Miskolic Mathematical Notes. 2013;14:323-333 
